Rev. salud pública. 14 (2): 213-225, 2012

\title{
Carga de enfermedad por cáncer en Santander, Colombia, 2005
}

\section{The burden of disease for cancer in Santander, Colombia, 2005}

Rafael Esquiaqui-Felipe ${ }^{1}$, Héctor Posso-Valencia ${ }^{2}$, Rolando E. Peñaloza ${ }^{2}$ y Jesús Rodríguez-García ${ }^{3}$

1 Facultad de Salud, Universidad Industrial de Santander. Bucaramanga, Colombia. esquiaqui22@hotmail.com

2 Centro de proyectos para el desarrollo (CENDEX). Universidad Pontificia Javeriana. Bogotá, Colombia.hposso@gmail.com; epenaloz@javeriana.edu.co

3 Facultad de Ciencias Económicas y Administrativas, Universidad Pontificia Javeriana. Bogotá, Colombia.rodriguez-j@javeriana.edu.co

Recibido 3 Junio 2011/Enviado para Modificación 14 febrero 2012/Aceptado 3 Abril 2012

\section{RESUMEN}

Objetivo Determinar la carga de la enfermedad por cáncer en Santander a través de los años de vida ajustados por discapacidad (AVISA).

Métodos Estudio descriptivo de carga de la enfermedad para determinar los AVISA producidos por Cáncer en Santander. La unidad de análisis fueron los registros del Observatorio Poblacional de Cáncer de la Universidad Autónoma de Bucaramanga y el registro de mortalidad del Departamento Administrativo Nacional de Estadísticas del año 2005.

Resultados La carga global de enfermedad para Santander se estimó en 877 AVISA totales por cada cien mil personas, de los cuales 50 eran debido a discapacidad y 827 a muerte prematura. Se evidencia que los AVISA por muerte prematura superan ampliamente a los AVISA por discapacidad, $94 \%$ versus 6 $\%$ respectivamente. En hombres los mayores valores de AVISAS por muerte prematura fueron estómago $(169,8)$, leucemias $(106,8)$, colon y recto $(100,4)$, linfomas y mielomas múltiples $(89,9)$. Mientras que en las mujeres los mayores valores se observaron en cérvico uterino $(122,4)$, mama $(121,9)$, estómago $(94,2)$ y leucemias $(86,0)$. Los tipos y las localizaciones de cáncer con más AVD fueron: colon y recto $(11,3)$, mama $(6,2)$, hígado $(6,05)$ y cérvico uterino $(5,43)$.

Conclusiones La carga de enfermedad por cáncer en Santander durante el año 2005 se debe principalmentea los años de vida perdidos por muerte prematura en ambos sexos y para todoslos grupos de edades.

Palabras Clave: Cáncer, costo efectividad, años de vida perdidos por discapacidad, prioridades en salud (fuente: DeCS, BIREME).

\section{ABSTRACT}

Objectives Determining the burden of cancer in Santander using disability adjusted life years (DALY). 
Methods This was a descriptive study of the disease burden for determining DALYs caused by cancer in Santander. The unit of analysis consisted of the Autonomous University of Bucaramanga's Population-Based Cancer Centre records and National Bureau of Statistics' mortality records for 2005.

Results The global burden of disease for Santander was estimated at 877 DALY per hundred thousand people, 50 being due to disability and 827 to premature death. It was seen that premature death DALY (94\%) greatly outweighed disability DALY (6\%). The highest DALY values due to premature death in men were related to cancer of the stomach (169.8), leukaemia (106.8), colon and the rectum (100.4), lymphomas and multiple myelomas (89.9), whilst the highest values in women were observed in cervical (122.4), breast (121.9) and stomach cancer (94.2) and leukaemia (86.0). The types and cancer sites related to more years of life lost (YLL) were the colon and rectum (11.3), breast (6.2), liver (6.05) and cervical cancer (5.43).

Conclusions The burden of disease from cancer in Santander in 2005 was mainly due to premature death YLL in both sexes and all age-groups.

Key Words: Cancer, priority, health, illness, burden (source: MeSH, NLM).

$\mathrm{L}$

a epidemiología de cáncer ha sido ampliamente estudiada, principalmente en términos de mortalidad. Pero el aumento de la sobrevida en muchos tumores malignos ha conllevado que cada vez más sea necesario tener en cuenta las consecuencias no mortales de la enfermedad (discapacidad debido a la propia enfermedad o al tratamiento, y el empeoramiento de la calidad de vida del paciente) (1).

Igualmente, el envejecimiento de la población es un fenómeno mundial, que hace referencia al proceso que asocia las tendencias de la mortalidad y las tasas de fecundidad en el transcurso del tiempo. En los últimos años en América se ha observado que las tasas de mortalidad y fecundidad han disminuido; mientras que la longevidad y la calidad del sistema de salud han aumentado, produciendo así una modificación en la estructura de edad de la población, con el incremento del peso relativo de los mayores (2).

Colombia es uno de los países de América Latina con transformaciones más notorias en su estructura poblacional, como consecuencia del acelerado proceso de transición demográfica, que ha ocasionado que las enfermedades crónicas reviertan mayor importancia en el contexto de la salud pública y en los gastos sanitarios (2).

Sin embargo, la evaluación del estado de salud de las poblaciones se ha basado tradicionalmente en indicadores simples (incidencia, mortalidad), 
los cuales pueden ser influidos por los problemas de salud de edades más avanzadas, que es donde ocurren la mayor cantidad de defunciones, lo que no permite evaluar el deterioro de la calidad de vida y discapacidad generada por enfermedades en la población, desagregadas por sexo y edad (3).

Estas limitaciones así como la focalización en sólo uno de los aspectos, han determinado la necesidad de desarrollar indicadores para medir otros aspectos del estado de salud, como el estado funcional (4).

Es por ello, que el estudio de la carga de enfermedad surgió como una alternativa para determinar la importancia relativa de los riesgos para la salud y sus consecuencias en los diferentes subgrupos de la población. Se distingue por la incorporación de datos sobre los resultados de salud que no conducen a la muerte en las medidas de resumen de la salud de las poblaciones (5).

Este tipo de estudio integra dos componentes fundamentales: los años de vida perdidos por muerte prematura (AVPM) y los años de vida vividos con discapacidad (AVD), permitiendo una perspectiva más amplia de calidad de vida de los individuos (6,7). Los años de vida ajustados por discapacidad (AVISA) evidencian las diferencias existentes entre la situación actual y la ideal de salud de una población $(6,7)$.

En Colombia se han realizado dos estudios de carga de enfermedad, el primero de ellos en 1994 y posteriormente en 2005 (8,9). En el ámbito latinoamericano, nacional y regional, Santander es el primero en utilizar esta metodología para determinar los AVISA producidos por Cáncer y comparar los resultados con estudios similares de otros países.

\section{MATERIALES Y MÉTODOS}

Se llevó a cabo un estudio descriptivo de la carga de enfermedad asociada con los diferentes tipos y localizaciones de cáncer en el año 2005 en Santander. A continuación se detallan los pasos seguidos hasta la obtención de los resultados.

Estimación de la población

Se obtuvo de los resultados del Censo del 2005, realizado por el Departamento Administración Nacional de Estadísticas (DANE) (10), y se construyeron tablas de poblaciones por grupos de edades $(0-4,5-14,15-29$, $30-44,45-59,60-69,70-79$ y $>80$ años) 
Estimación de la mortalidad

Los datos de mortalidad se obtuvieron del DANE (10). A partir de las causas básicas de muertes codificadas según Clasificación Internacional de Enfermedades de la Organización Mundial de la Salud, en su décima versión, se procedió a agruparlas en un listado de causas por grupo de edad y sexo. Las causas estudiadas fueron (cáncer cérvico uterino C53, cáncer de esófago $\mathrm{C} 15$, cáncer de estómago $\mathrm{C} 16$, cáncer de colon y recto C18-C21, cáncer de hígado C22, cáncer de vías biliares C23-C24, cáncer de páncreas $\mathrm{C} 25$, cáncer de tráquea, bronquios y pulmón C33-C34, melanoma y otros canceres de piel C43-C44, cáncer de mama C50, cáncer de cuerpo del útero C54, cáncer de ovario C56, cáncer de próstata C61, cáncer de vejiga $\mathrm{C} 67$, cáncer de encéfalo C71, linfoma y mieloma múltiple C81-C90, C96 y leucemia C91- C95 y otros tumores malignos C17, C26, C30-C32, C37-C41, C45-C49, C51-C52, C57-C58, C60, C62-C66,C68-C70,C72-C75, C97).

Por otro lado, las tablas de defunciones construidas fueron sometidas a un algoritmo (11) que permitió reclasificar algunas causas que no correspondían a causas básicas de muerte, obteniéndose así unas tablas de defunciones más consistentes, que posteriormente fueron ajustadas por cobertura del registro de defunciones.

Estimación de la morbilidad

Las estimaciones de las incidencias de los cánceres específicos partieron de los datos por edad, sexo y causas, suministrada por el Registro Poblacional de Cáncer del Área Metropolitana de Bucaramanga (12) mientras que las estimaciones de prevalencias se calcularon por el método propuesto por Pisani 2.002(13).

Asignación de la discapacidad

Se utilizaron las tablas de secuelas y discapacidad atribuible a cada enfermedad según los cálculos provistos por Murray \& López (14).

Cálculo de los años de vida perdidos por muerte prematura (AVPM) y los años de vida perdidos por discapacidad (AVD)

Para calcular estos dos indicadores se utilizó la tabla de esperanza de vida japonesa, ajustada con descuento del $3 \%$ y ponderada desigual por edades ( $\mathrm{B}=0,04$ y $\mathrm{C}=0,1658)$, publicada por Murray (15), y las salidas procedentes 
de la utilización del Dismod (casos incidentes, duración y la edad de inicio), las cuales garantizan una adecuada consistencia entre los datos $(16,17)$.

Como en el presente estudio no se contaba con información del estadio clínico de cada uno de los casos y para no subestimar la carga por discapacidad, se asumió que los individuos con cáncer se encontraban en el estadio terminal. La carga de enfermedad total, se dio en términos de AVISA por 100000 habitantes, se calculó como la suma de los AVPM y de AVD.

\section{RESULTADOS}

La carga global de enfermedad se estimó para Santander en el año 2005 en 877 AVISA totales por 100000 habitantes, 50 AVISA atribuibles a discapacidad por 100000 habitantes y 827 AVISA de mortalidad por 100000 habitantes.

En la Figura 1 se pueden observar los pesos relativos que tienen los AVPM y AVD en la carga global. Los AVPM superan ampliamente, 94 $\%$ versus $6 \%$ a los AVD. Por sexo, entre las mujeres, la diferencia es ligeramente mayor pues el $6 \%$ de los AVISA totales corresponden a AVD, mientras que en hombres esta proporción desciende a $5 \%$.

Figura 1. Pesos relativos de AVPM y AVD ( \%) por sexo, Carga de Enfermedad Santander - Cáncer 2005

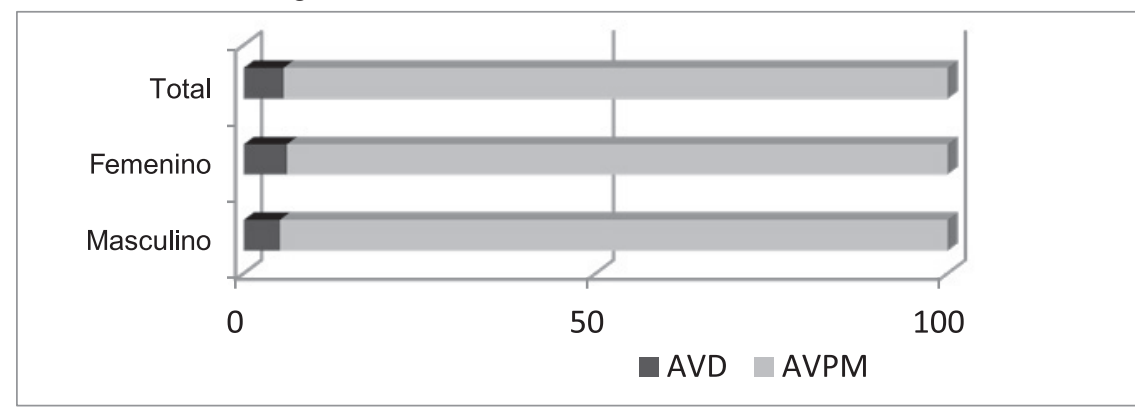

La distribución de AVD y AVPM por grupos de edad y sexo, presenta los perfiles que se muestran en las Figuras 2 y 3 , para hombres y mujeres. Resultan ser diferentes los perfiles de distribución de la carga entre géneros, observándose en los hombres el valor máximo de los AVISA de discapacidad en el grupo de 5-14 años. Mientras que en la mujeres ocurre en el grupo 15-29 años. 
En la Tabla 1 se observan las principales causas de AVISA en hombres y mujeres de todas las edades. En hombres de todas las edades se pierden 839 AVISA Totales por 100000 hombres (5,20\% de ellos por AVD). En mujeres por su parte las principales causas de AVISA para todas las edades, generan una pérdida de 914 AVISA totales por 100000 personas de los cuales el $94 \%$ son debidos a AVPM.

Figura 2. Pesos relativos de AVPM y AVD ( \%) por edad, sexo masculino. Santander, Carga de Enfermedad- Cáncer, 2005

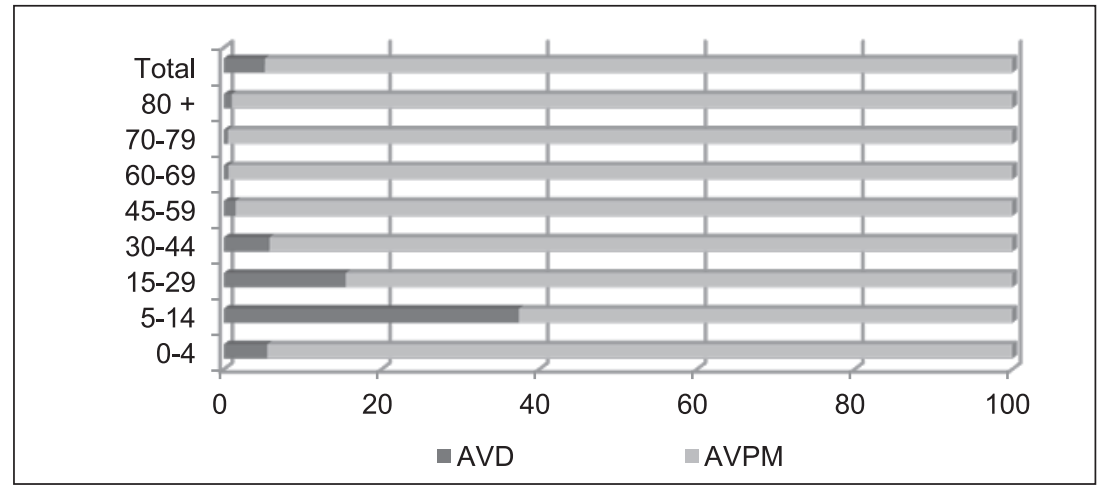

Figura 3. Pesos relativos AVPM y AVD ( \%) por edad, sexo femenino. Santander, Carga de Enfermedad- Cáncer, 2005

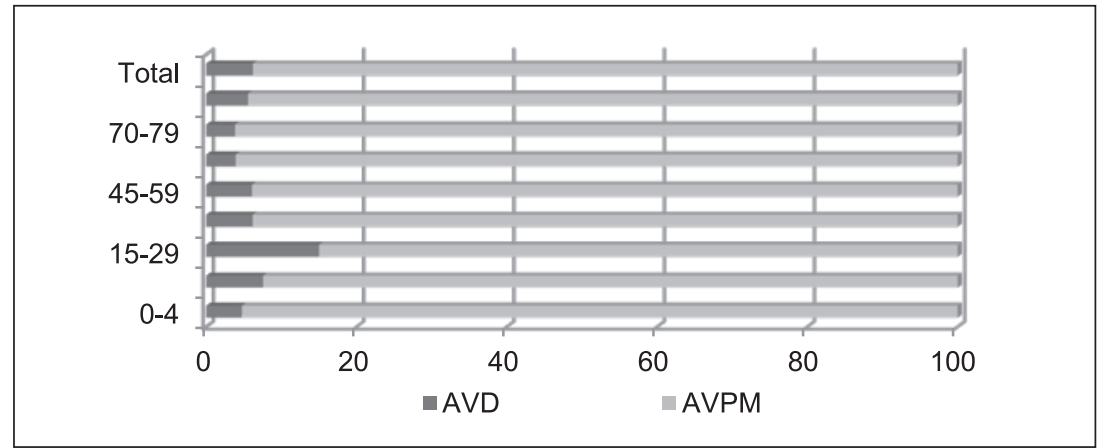

En la población masculina el cáncer de estómago es la primera causa de AVISA totales, seguido por las leucemias con 106 AVISA totales y el cáncer de colon y recto con 100 AVISA totales por 100000 habitantes. Mientras que el cáncer de próstata ocupa el séptimo lugar con 53 AVISA totales por 100000 habitantes. 


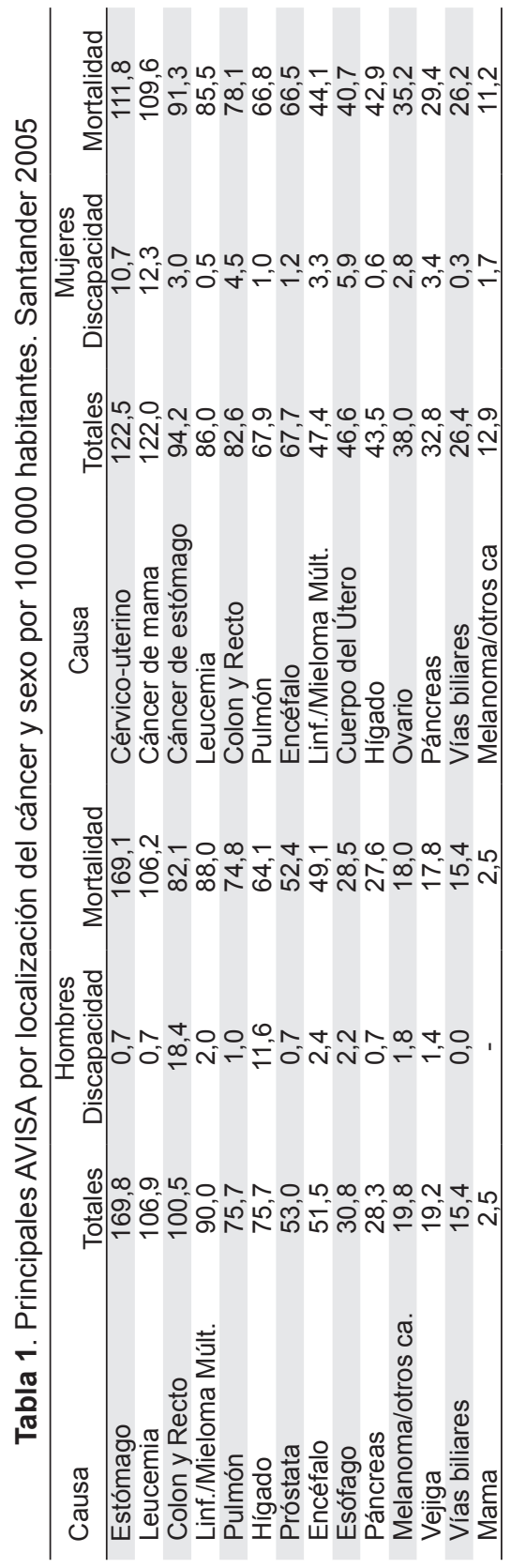


Mientras que en la población femenina el cáncer cérvico uterino ocupa el primer lugar con 122 AVISA totales por 100000 habitantes, seguido por el cáncer de mama con 121 AVISA totales por 100000 habitantes y el cáncer de estómago con 94 AVISA totales por 100000 habitantes.

A continuación se muestra en la Tabla 2 la carga global de enfermedad por causas y edades en ambos sexos. En Santander se pierden 877 AVISA totales por 100000 personas, el 5,7\% de los cuales se deben a pérdidas por discapacidad. Se observa que el cáncer de estomago se presenta como la principal causa de años saludables perdidos con 131 AVISA totales por 100000 personas ( $15 \%$ de los AVISA totales). Por su parte, los trastornos hematológicos son responsables del $19 \%$ de la carga global de AVISA por 100000 personas y el cáncer de colon y recto es responsable del 22,6 \% de los AVD en Santander.

Tabla 2. Principales AVISA por localización del Cáncer en ambos sexos, Carga de Enfermedad. Santander 2005

\begin{tabular}{clccc}
\hline Orden & \multicolumn{1}{c}{ Causa } & $\begin{array}{c}\text { AVISA Totales } \\
\text { x 100 000 } \\
\text { personas }\end{array}$ & $\begin{array}{c}\text { AVD x } \\
100 \text { 000 } \\
\text { personas }\end{array}$ & $\begin{array}{c}\text { AVPM x 100 000 } \\
\text { personas }\end{array}$ \\
\hline 1 & Estómago & 131,5 & 1,8 & 129,7 \\
2 & Leucemia & 96,3 & 0,6 & 95,7 \\
3 & Colon y Recto & 91,5 & 11,4 & 80,1 \\
4 & Pulmón & 71,7 & 1,0 & 70,7 \\
5 & Linfoma y Mieloma Múltiple & 68,4 & 2,7 & 65,8 \\
6 & Mama & 63,1 & 6,3 & 56,8 \\
7 & Cérvico-uterino & 62,1 & 5,4 & 56,6 \\
8 & Encéfalo & 59,7 & 1,8 & 57,9 \\
9 & Hígado & 59,4 & 6,1 & 53,3 \\
10 & Páncreas & 30,6 & 2,1 & 28,5 \\
11 & Próstata & 26,2 & 0,3 & 25,9 \\
12 & Cuerpo del Útero & 23,6 & 3,0 & 20,6 \\
13 & Vías Biliares & 21,0 & 0,2 & 19,6 \\
14 & Esófago & 20,9 & 1,3 & 17,8 \\
15 & Ovario & 19,2 & 1,4 & 14,6 \\
\hline 16 & Melanoma/otros Ca. de Piel & 16,3 & 1,8 & 12,7 \\
\hline 17 & Vejiga & 15,9 & 3,3 & \\
\hline
\end{tabular}

\section{DISCUSIÓN}

En mujeres, los tipos y las localizaciones de cáncer que provocaron mayores AVISA totales fueron los siguientes: cáncer cérvico uterino, el cáncer de mama, cáncer de estómago, leucemias y el cáncer de colon y recto. Las diferencias entre hombres y mujeres fueron menores en la leucemia y el linfoma que en el resto de los tipos y localizaciones de cáncer estudiados. 
En hombres, el cáncer de pulmón se encuentra en el quinto lugar con 76 avisas totales perdidos por 100000 hombres, mientras que el cáncer de hígado y de próstata, en los lugares sexto y séptimo, contribuyen con 15 $\%$ del total de AVISA por 100000 hombres entre ambas. Por otro lado, el cáncer de estómago, las leucemias, el cáncer de colon y recto y el linfoma y mieloma múltiple suman casi la mitad del total de años saludables perdidos, a expensas fundamentalmente de AVPM.

Comparación con otros estudios de carga de enfermedad

En el ámbito nacional se han realizado tres estudios de carga de enfermedad, el primero de ellos fue en el año 1994 por el Ministerio de Salud, el segundo fue realizado por investigadores del Ministerio de Salud y que fue publicado en el año 2000 (18) y el último de estos realizado en el año 2005 por la Universidad Javeriana en conjunto con el Ministerio de Protección Social $(8,9)$.

Sin embargo, dichos estudios utilizaron metodologías distintas, que hacen difícil de comparar estos estudios entre sí, por ejemplo el estudio de 1994 realizó estimaciones directas de AVPM e indirectas de AVD, mientras que el estudio publicado en el año 2000, analizó la carga global de enfermedad en Colombia a través de estimaciones directas de AVPM y AVD, pero estas últimas solo se calcularon para causas de mortalidad mayor a 0 .

Por el contrario, al comparar los resultados del grupo II del estudio de carga enfermedad del año 2005 realizado por Cendex de la Universidad Javeriana con los resultados del estudio santandereano, se observó que los AVISA por cáncer eran muy similares (998 AVISA versus 877 AVISA) y los porcentaje de AVPM y AVD eran los mismos (96 \% y 4 $\%$ respectivamente). Cabe precisar que en el ámbito internacional se han realizado 2 estudios de carga de enfermedad por cáncer, uno de ellos en España en el año 2000 (19) y otro en la Habana Cuba en el año 2002 (20). Este último por diferencias metodológicas no permite la comparación con el estudio en Santander.

En la Tabla 3, se muestran los indicadores construidos con los resultados de estimar la carga de enfermedad en los estudios seleccionados y se pueden apreciar diferencias en las fuentes de los pesos de discapacidad utilizados en los diferentes estudios. 
Tabla 3. Valores de indicadores de AVISAS y herramientas metodológicas empleadas según estudio y tipo de indicador

\begin{tabular}{|c|c|c|c|c|}
\hline Indicador & España 2000 & $\begin{array}{c}\text { Cuba } \\
1990-2002\end{array}$ & $\begin{array}{l}\text { Colombia } \\
\text { (cáncer) } \\
2005\end{array}$ & $\begin{array}{l}\text { Santander } \\
2005\end{array}$ \\
\hline $\begin{array}{l}\text { AVISAs } \\
\text { totales/100.000 } \\
\text { personas }\end{array}$ & 828.997 & ND & 998 & 877 \\
\hline AVD / 100.000 & 130.726 & ND & 62 & 50 \\
\hline AVPM por 100.000 & 698.271 & ND & 936 & 827 \\
\hline AVPM/AVD & 5.3 & ND & 15,0 & 16,5 \\
\hline Masculino/Femenino & ND & ND & 0,9 & 0,9 \\
\hline $\begin{array}{l}\text { Esperanza de vida } \\
\text { total al nacer (años) }\end{array}$ & 85 & ND & 73,3 & 73,3 \\
\hline $\begin{array}{l}\text { Fuente de } \\
\text { esperanzas de vida } \\
\text { de referencia para el } \\
\text { cálculo de AVPM }\end{array}$ & $\begin{array}{l}\text { Murray \& } \\
\text { López, } 1994\end{array}$ & $\begin{array}{c}\text { Oficina Nacional } \\
\text { de Estadística } \\
\text { Cuba }\end{array}$ & $\begin{array}{l}\text { Murray \& } \\
\text { López, } 1996\end{array}$ & $\begin{array}{c}\text { Murray \& } \\
\text { López, } 1996\end{array}$ \\
\hline $\begin{array}{l}\text { Ponderación de } \\
\text { edad }\end{array}$ & Uniforme & ND & Exponencial & Exponencial \\
\hline Tasa de descuento & $3 \%$ & ND & $3 \%$ & $3 \%$ \\
\hline $\begin{array}{l}\text { Fuente de pesos de } \\
\text { discapacidad }\end{array}$ & $\begin{array}{c}\text { Murray \& López, } \\
\text { 1996, Stuthard } \\
\text { et al, } 1997\end{array}$ & $\begin{array}{c}\text { Murray \& } \\
\text { López, } 1996\end{array}$ & $\begin{array}{c}\text { Murray \& } \\
\text { López, } 1996 \text { y } \\
\text { ajustes locales }\end{array}$ & $\begin{array}{c}\text { Murray \& } \\
\text { López, } 1996 \text { y } \\
\text { ajustes locales }\end{array}$ \\
\hline
\end{tabular}

En el estudio cubano no mencionan los resultados de la carga total por cáncer, ni tampoco los aspectos metodológicos como son los AVD, AVPM, tasas de descuentos, ni valor de esperanza total de vida al nacer, solo mencionan que los datos de esperanza de vida fueron tomados del Instituto Nacional de Estadísticas de Cuba del año 1990. La única de referencia es haber tenido en cuenta como fuente de discapacidad las tablas de Murray \& López.

No hay diferencias entre el estudio español y el estudio santandereano con respecto a la selección de la tabla de esperanzas de vida de referencia ni en la cifra empleada como tasa de descuento para el cálculo de los AVPM.

En conclusión la carga de enfermedad por cáncer en Santander durante el año 2005 se debe mayoritariamente a los años de vida perdidos por muerte prematura (94\%). Por otra parte, se pudo apreciar que en la población 0-4 años las leucemias, linfomas y mielomas múltiples producen una alta carga de enfermedad con 131,8 AVISA a expensas de los AVPM (81\%). Esta alta mortalidad es producida por múltiples factores como lo son: el acceso tardío a atención médica primaria y terciaria, el diagnóstico erróneo por parte de los profesionales de la salud e inadecuada infraestructura 
de gestión de datos, que impiden dar a estos niños y adolescentes un tratamiento oportuno.

Por ello, se hace necesario que los médicos santandereanos sospechen de cáncer en menores de 5 años, al igual que lo hacen con las otras patologías específicas de la infancia, ya que un $70 \%$ de los niños diagnosticados y tratados oportunamente se curan (21).

Asimismo, se debe considerar prioritario intervenir los grupo de 5 a 14 años en hombres y el grupo de 16 a 29 años en mujeres para disminuir los AVD generados por esta patología. Además es ineludibleimplementar estrategias de educación y nutrición en la población para disminuir la mortalidad por cáncer de estómago a largo plazo.

La principal limitación con que se enfrenta este estudio es la escasa disponibilidad de estudios de prevalencias y de sobrevida en Cáncer. Además de las dificultades a las que se enfrentan los registros poblacionales para la obtención de la información de las diferentes entidades de salud en la región, situación que se ha resuelto sólo en parte calculando estos indicadores a partir de métodos internacionales (13) y extrapolando otros a partir de poblaciones que presentan en principio un patrón epidemiológico semejante al nuestro (similar sobrevida general en niños y adultos).

Los AVISA han sido criticados por incorporar preferencias sociales que pueden no ser universalmente aceptadas: la cuantía de los AVPM depende del límite de esperanza de vida elegido y los AVD dependen en gran medida del peso de la discapacidad atribuido a las diferentes enfermedades, establecido mediante paneles de expertos. Igualmente se ha cuestionado la incorporación de tasas de descuento y pesos por edades al cálculo de los AVISA. Aun considerando estas críticas, es evidente que la elevada discapacidad que causan las enfermedades más prevalentes en nuestro país debe tenerse en cuenta a la hora de analizar y vigilar la salud, priorizar intervenciones y distribuir los recursos entre los diferentes programas de salud dirigidos a mejorar la salud de la población infantil. Los AVISA permiten comparar problemas de salud que por sus diferentes características epidemiológicas y clínicas (incidencia, duración, discapacidad y mortalidad) difícilmente pueden equipararse

Agradecimientos: Al Registro Poblacional de Cáncer del Área Metropo-litana de 
Bucaramanga por su apoyo en el suministro de las bases de datos de incidencias y al Centro de Proyecto para el Desarrollo (Cendex) de la Universidad Javeriana por sus aportes y soportes metodológicos.

\section{REFERENCIAS}

1. World Health Organization. [Internet]. Disponible en: www.who.int/cancer/publicat/ WHOCancerBrochure2007.FINALweb.pdf.Consultado Septiembre del 2010.

2. Departamento Nacional de Planeación. Envejecimiento y vejez (Documento CONPES 2793). Santafé de Bogotá D.C., Colombia: DNP; 2010.

3. Organización Panamericana de la Salud. Técnica para la medición del impacto de la mortalidad: Años potenciales de vida perdidos. Bol Epidemiol. 2003; 24:1-16.

4. Ministerio de salud. [Internet]. Estudio de carga de enfermedad y carga atribuible Chile, 2007. Disponible en: http://epi.minsal.cl/epi/html/invest/estudios.htm. Consultado: 2010, 4 de octubre.

5. García JF, Royo MA (Ed.). Manuales de dirección médica y gestión clínica. Salud pública y Epidemiología. Madrid: Díaz de Santos S.A; 2006.

6. World Health Organization. [Internet]. Disponible en:http://www.who.int/healthinfo/gbd 2000guidelines.pdf Consultado 22 Sept. 2010.

7. López AD, Mathers CD, Ezzati M, Jamison DT, Murray JL [Internet]. La medición de la carga mundial de morbilidad y de los factores de riesgo 1990-2001. Disponible en: http://files.dcp2.org/pdf/GBD/GBDSpanish1.pdf Consultado: 2010, 22 de Septiembre.

8. Escobar ML, Gallardo HM, Giraldo PG, Londoño JL y Rodríguez J. La carga de la enfermedad en Colombia. Bogotá D.C: Carrera Séptima Ltda; 1994.

9. Acosta N, Peñalosa E, Rodríguez J [Internet]. Carga de enfermedad Colombia 2005. Disponible en: http://www.cendex.org.co/GPES/informes/PresentacionCarga_Informe. pdf Consultado: 2010, 22 de Septiembre.

10. DepartamentoAdministrativo Nacional deEstadística. [Internet]. Disponibleen:www.dane.gov. co/index.php?option=com_content\&task=category\&sectionid=16\&id=497\&ltemid=995. Consultado: Septiembre de 2010.

11. Rodríguez J, Ruiz F, Peñalosa E. Desarrollo e implantación de la metodología de Esperanza de Vida Saludable (EVISA). Rev. Gerenc. Polit. Salud. 2009; 8:123-139.

12. Sánchez C, Villamizar L. Mortalidad por tumores en Santander, 1998-2006. Med UNAB. 2008; 11 (2): 83-94.

13. Pisani P, Bray F, Maxwell P. Estimates of the world-wide prevalence of Cancer for 25 sites in the adult population. Int J. Cancer. 2002; 97 Supl 1:72-81.

14. Murray C, Lopez A. The global burden of disease. Global burden of disease and injury series. Vol I. Cambridge: Harvard School of Public Health. Harvard University Press; 1996. p.412-16.

15. Murray C, López A. Global health statistics. Global burden of disease and injury series. Vol II. Cambridge: Harvard School of Public Health. Harvard University Press; 1996.

16. Dismod II. Programa desarrollado por la Organización Mundial de la Salud [programa de ordenador]. Versión 1.04.University of Queensland: School of Population Health 2001-12008.

17. Murray C, López A. The global burden of disease. The global burden of disease. Global burden of disease and injury series. Vol I. World Health Organization; 1996.

18. Rodríguez J, Gallardo H. Carga Global de Enfermedad. Colombia 1985-1995. Bogotá D.C.: Ministerio de la Protección Social; 1999.

19. Génova R, Álvarez M, Morant MC. Estimación de la Carga de Enfermedad en España 
en el año 2000. Cuadernos geográficos de la Universidad de Granada. 2005; (1) 36:571-576.

20. Alonso E, Seuc Jo A, Galán Y. La carga de enfermedad por cáncer en Cuba en el período 1990-2002. Pan American Journal of PublicHealth 2009; 26(5): 412-418.

21. Bravo LE, Collazos T, García LS, Gutiérrez A, Carrascal E. Cáncer infantil en Cali, Colombia 1994-2003. Registro Poblacional de Cáncer en Cali, 2009. Cali, Colombia: Catorse; 2009. 